\title{
Graduate Student Award Finalists to Compete at the 1997 MRS Spring Meeting
}

Nine finalists will compete for the MRS Graduate Student Awards to be presented Monday, March 31, at 6:00 p.m., in the San Francisco Marriott Hotel, Salon 7. Each finalist will give a 10-minute presentation during the Graduate Student Award Special Talk Session. The schedule will be provided on-site.

The finalists, all authors or co-authors of papers to be presented at the 1997 MRS Spring Meeting, were selected on the basis of the quality and thoroughness of their research, the originality and independence of their contributions, and their promise for future achievements in materials research.

Following is the list of finalists, their places of study, titles of papers, and the symposium or symposia in which the finalists are participating.
Graduate Student Award Finalists1997 MRS Spring Meeting

Loren Chow, University of California-Los Angeles, "The Processing and Characterization of Hybrid Silica-Based Xerogel Films" (Symposium N)

Ali Firouzi, University of California-Santa Barbara, "Magnetic-Field-Induced Orientational Ordering of Silicate-Surfactant Mesophases" (Symposium V)

Gillian Goward, University of Waterloo, "H-2 and Li-7 Solid State NMR Studies of Conducting Polymer/Oxide Nanocomposites (Symposium V)

Joohyun Koh, The Pennsylvania State University, "Structural and Optical Properties of $\mu \mathrm{c}-\mathrm{Si}: \mathrm{H}:(\mathrm{P}, \mathrm{B}) / \mathrm{a}-\mathrm{Si}: \mathrm{H}$ Junctions in a-Si:H-Based Solar Cells" and "Characterization of MonolayerLevel Composition and Optical Gap Profiles in Amorphous Silicon-Carbon Alloy BandgapModulated Structures" (Symposium A)

Shouliang Lai, University of Illinois-Urbana,
"Melting Point Depression of Ultra-Thin Discontinuous Al Films" (Symposium K)

Eric B. McDaniel, University of Virginia, "Measurement of Strain Associated with Defects in $\mathrm{SrTiO}_{3}$ Bicrystal Substrates Using Near Field Scanning Optical Microscopy" and "Microstructure Defects in $\mathrm{SrTiO}_{3}$ Bicrystals and Their Influence on $\mathrm{YBa}_{2} \mathrm{Cu}_{3} \mathrm{O}_{7}$ Grain Boundary Junctions" (Symposium L)

Eric A. Stach, University of Virginia, "Measurement of Misfit Dislocation Propagation Velocities During UHV-CVD Growth of SiGe/ $\mathrm{Si}$ (100)" (Symposium B)

Blair Tuttle, University of Illinois-Urbana, "Vibrational Signatures of $\mathrm{H}$ in Amorphous Silicon" (Symposium A)

Chung-chih Wu, Princeton University, "Effects of Plasma Treatment of the Anode Surface on the Performance of Organic Light Emitting Devices" and "Integrated Organic Light Emitting Diode Structures Using Single-Layer Doped Polymer Thin Films" (Symposium H)

\section{NEW Gordon Research Conference}

\section{Materials Processes Far from}

\section{Equilibrium}

\section{Aug. 17-22, 1997, Kimball Union, NH}

\section{Session Topics}

\begin{tabular}{|c|c|}
\hline $\begin{array}{l}\text { - Implantation } \\
\text { - Modeling } \\
\text { - Phase formation }\end{array}$ & $\begin{array}{l}\text { - Defect formation } \\
\text { - Surface stimulation } \\
\text { - Future challenges }\end{array}$ \\
\hline
\end{tabular}

+ Poster sessions

\section{Conference chairs:}

\section{Eric Chason}

Sandia National Laboratory

Albuquerque, NM

ph: 505-844-8951;

ehchaso@sandia.gov
Tomas Diaz de la Rubia

Lawrence Livermore

National Laboratory

ph: 510-422-6714;

delarubia@llnl.gov
For more information, contact:

\section{Gordon Research Center}

U. of Rhode Island, PO Box 984, West Kingston, RI 02892-0984

phone: 401-783-4011 FAX: 401-783-7644

E-MAIL: GRC@GRCMAIL.GRC.URI.EDU

\section{(MO)CVD of Oxide Films}

\section{Custom Film Services}

Transparent Conductive Oxides

(ITO, ZnO, ........)

Luminescent Oxides (including rare earth doping)

$\left(\mathrm{ZnO}, \mathrm{ZnGa}_{2} \mathrm{O}_{4}, \mathrm{ZnSiO}_{4}, \mathrm{Y}_{2} \mathrm{O}_{3} \ldots \ldots ..\right)$

Epitaxy

$\left(\mathrm{ZnO}, \mathrm{MgO}, \mathrm{Al}_{2} \mathrm{O}_{3} \ldots \ldots ..\right)$

Others upon request

(Dielectrics, Ferroelectrics, Piezoelectrics,

Superconductors, Magnetoresistive; $\mathrm{TiO}_{2}, \mathrm{CuO}$,

$\left.\mathrm{Ba}_{2} \mathrm{O}_{3}, \mathrm{YBCO}, \mathrm{Sr}_{2} \mathrm{O}_{3}, \mathrm{CeO}_{2} \ldots \ldots.\right)$

\section{Custom Oxide Deposition Consulting}

- We will work with you to optimize oxide films for your applications SMI

\section{STRUCTURED MATERIALS INDUSTRIES,} INCORPORATED

120 Centennial Avenue, Piscatoway, NJ 08854-3908

Ph: (908) 885-5909; Fax: (908) 885-5910

e-mail: 75031.1321@Compuserve.com 